\title{
Mejora del desempeño ambiental y energético de la vivienda de interés prioritario en Medellín con el uso de ladrillos cerámicos modificados*
}

\author{
Gloria Restrepo-Zapata* \\ Carlos Cadavid-Restrepo ${ }^{* * *}$
}

\author{
Recibido: 06/16/2017 - Aceptado: 28/11/2018 \\ https://doi.org/10.22395/rium.v18n35a3
}

\begin{abstract}
Resumen
La vivienda es un elemento que atiende funciones sociales, culturales, de seguridad, salud y bienestar de los individuos; el Estado es el responsable de garantizar el acceso de la población a esta. La construcción, operación y demolición de las viviendas genera impactos ambientales significativos que reducen su desempeño ambiental y energético. Dicho desempeño se podría optimizar a lo largo de su ciclo de vida con la adopción de elementos de construcción sostenible, que en el contexto de la vivienda de interés prioritario de Medellín, comienzan a ser una demanda de los habitantes de las viviendas por los ahorros en costos de servicios públicos, confort térmico y calidad de vida. Este artículo de investigación demuestra que el uso de ladrillos modificados constituye una oportunidad para minimizar el consumo de recursos naturales no renovables -como la arcilla y el carbón-empleados tradicionalmente en la fabricación de vivienda, así como para recuperar, reciclar y reintegrar recursos alternativos provenientes de fuentes secundarias, como los residuos sólidos urbanos. Se demostró que es posible, contribuir no solo a la disminución de la huella de carbono de la vivienda de interés prioritario, sino también a la generación de un conjunto de externalidades, entre las que destacan la innovación y desarrollo tecnológico, nuevos procesos industriales y modelos de negocio, mayor rentabilidad y competitividad empresarial, responsabilidad social, disminución en la generación de impactos ambientales - como la contaminación del aire y en los efectos asociados a la salud pública.
\end{abstract}

Palabras clave: ladrillo cerámico modificado; combustibles sólidos recuperados; vivienda de interés prioritario; desempeño ambiental; desempeño energético; eficiencia de recursos; análisis de ciclo de vida; externalidades.

* Este artículo surge de la investigación Mejoramiento del desempeño ambiental y energético de la vivienda de interés prioritario en Medellín, a través del uso de materiales de construcción modificados, desarrollado entre el 2016 y el 2017 y financiado con recursos propios.

** Ingeniera ambiental, especialista en Manejo de Recursos Naturales y magíster en Ciencias Ambientales: Sostenibilidad, con experiencia en economía circular, ecología urbana, producción y consumo sostenible. Correo electrónico: glorestrepoz@gmail.com. Orcid: https://orcid.org/0000-0003-0610-7418

*** Ingeniero químico, especialista en Ingeniería Ambiental, especialista en Gerencia del Ambiente, magíster en Eco auditorias y Planeación Ambiental Empresarial, con experiencia en economía sostenible, contabilidad ambiental, eficiencia de recursos y producción y consumo sostenible. Correo electrónico: carlosfcadavidr@ outlook.com. Orcid: https://orcid.org/0000-0002-9027-2856 


\title{
Improvement of Environmental and Energetic Performance of Social Housing in Medellin by Using Modified Ceramic Bricks
}

\begin{abstract}
Housing seeks to cover people's basic needs by attending to social, cultural, security, health and wellbeing functions. The State is responsible of ensuring access to housing. Construction, operation and demolition of houses generates significant environmental impacts that reduce environmental and energetic performance. Performance could be optimized along the life-cycle of the construction by adopting principles of sustainable construction, which in the context of the low-income housing of Medellin, have begun to be demanded by their inhabitants as a matter of savings for public services, thermal comfort and quality of life. This research article shows that the use of modified ceramic brick is an opportunity to minimize consumption of nonrenewable natural resources such as clay and coal-traditionally used in housing manufacture, as well as to recover, recycle and reintegrate alternative resources from secondary sources such as urban solid wastes. This does not only reduce carbon footprint of the low-income houses, but also generates externalities which stand out in innovation and technological development, new industrial processes and business models, greater profitability and enterprise competitiveness, social responsibility, minimization of environmental impacts such as air pollution, and effects on public health that are associated.
\end{abstract}

Keywords: modified ceramic brick; solid recovered fuels; low-income housing; environmental performance; energy performance; resource efficiency; life-cycle assessment; externalities.

\section{Melhoria do desempenho ambiental e energético $d$ a habitação de interesse social em medellín com o uso de tijolos cerâmicos modificado}

\begin{abstract}
Resumo
A moradia é um elemento que atende funções sociais, culturais, de segurança, saúde e bem-estar dos indivíduos; o Estado é o responsável por garantir o acesso à moradia para a população. A construção, a operação e a demolição das habitações geram impactos ambientais significativos que reduzem seu desempenho ambiental e energético. Tal desempenho poderia ser otimizado ao longo de seu ciclo de vida com a adoção de elementos de construção sustentável que, no contexto da habitação de interesse social em Medellín, começa a ser uma demanda dos habitantes por causa da economia nos gastos com serviços públicos, da comodidade térmica e da qualidade de vida. Este artigo de pesquisa mostra que o uso de tijolos modificados constitui uma oportunidade para minimizar o consumo de recursos naturais não renováveis - como a argila e o carbono - tradicionalmente empregados na fabricação de habitações, assim como para recuperar, reciclar e reintegrar recursos alternativos provenientes de fontes secundárias, como os resíduos sólidos urbanos. Demonstrou-se que é possível contribuir não apenas com a diminuição da emissão de carbono da habitação de interesse social, mas também com a geração de um conjunto de externalidades, entre as quais se destacam a inovação e o desenvolvimento tecnológico, novos processos industriais e modelos de negócio, maior rentabilidade e competitividade empresarial, responsabilidade social, diminuição na geração de impactos ambientais — como a contaminação do ar e nos efeitos associados à saúde pública.
\end{abstract}

Palavras-chave: tijolo cerâmico modificado; combustíveis sólidos recuperados; habitação de interesse social; desempenho ambiental; desempenho energético; eficiência de recursos; análise de ciclo de vida; externalidades. 


\section{INTRODUCCIÓN}

Más que una necesidad, la vivienda constituye un elemento para satisfacer necesidades básicas de la población [1], ya que hace posible atender funciones vitales, sociales y culturales de las personas y sus familias, a la vez que proporciona abrigo, resguardo y reposo. Sin embargo, en muchos casos, se caracteriza por presentar un alto costo al que familias o individuos con restricciones de ingresos económicos, enfrentados al desempleo o al subempleo, difícilmente tienen acceso, por lo cual se convierten en una responsabilidad de la sociedad [2]. Para dar cumplimiento a tal responsabilidad, los gobiernos han adoptado políticas que promueven el desarrollo de proyectos de viviendas de bajo costo que cumplan con condiciones básicas de calidad y habitabilidad, en las cuales se integran pocos o ningún concepto de sostenibilidad, tales la como eficiencia energética y el uso eficiente de los recursos [3].

En Colombia, como medida para reducir el déficit habitacional y favorecer a la población de escasos recursos, el gobierno nacional, según el decreto 0075 [4], ha creado dos figuras de vivienda social: Vivienda de Interés Social (VIS), definida como la "unidad habitacional que cumple con los estándares de calidad en diseño urbanístico, arquitectónico y de construcción y cuyo valor no exceda ciento treinta y cinco salarios mínimos mensuales legales vigentes (135 SMMLV)"; y Vivienda de Interés Prioritario (VIP), entendida como la "vivienda cuyo valor máximo será de setenta (70) SMMLV". En dicho decreto se establecieron y regularon algunos de los lineamientos técnicos y habitacionales con los cuales deberán cumplir, mínimamente, tales viviendas; así como se han desarrollado guías técnicas que establecen parámetros para la formulación, ejecución y puesta en marcha de dichos proyectos con el objetivo de disminuir el déficit cuantitativo de la vivienda en el país [5].

La ciudad de Medellín y el Valle de Aburrá, como su área de influencia, han considerado la promoción de la vivienda social como un instrumento para subsanar el déficit cualitativo y cuantitativo de la región, que además de que provea albergue y protección a la población, pueda adoptar criterios de sostenibilidad que contribuyan a la consolidación y construcción de un territorio sostenible [6]. Según el Plan Estratégico Habitacional de Medellín, Pehmed 2020 [7-8], el $48 \%$ del parque habitacional de Medellín corresponde a los estratos socioeconómicos 1 y 2 ; quienes a su vez presentan el mayor déficit cualitativo y cuantitativo habitacional de la ciudad.

Un aspecto importante asociado con la sostenibilidad de la vivienda es aquel relacionado con el consumo de energía dado a lo largo de su ciclo de vida; el cual supone principalmente los materiales y productos de construcción empleados. Esta condición deriva por lo tanto en una aportación significativa de emisiones de gases de efecto invernadero provenientes de la vivienda durante su ciclo de vida. 
La construcción de VIP en Medellín enfrenta importantes retos para el desarrollo de un territorio más sostenible [9-10] puesto que las edificaciones contribuyen con cerca del $38 \%$ de la generación global anual de gases de efecto invernadero y consumen más del $40 \%$ de la energía generada en países desarrollados y en vía de desarrollo.

\section{LA VIP EN EL CONTEXTO DE LA CONSTRUCCIÓN SOSTENIBLE}

Desde hace más de cuatro décadas, los procesos de construcción de vivienda estuvieron enfocados en garantizar una buena calidad de la edificación y en el control de los costos [11]. Sin embargo, el creciente interés por la protección del medio ambiente y la adopción de elementos que promuevan el desarrollo sostenible han causado impactos en las formas de construcción, lo cual dio paso al concepto de construcción sostenible que, según el Área Metropolitana del Valle de Aburrá es:

[...] un proceso integral que busca contribuir al incremento de la calidad de vida de la población; así como al desarrollo económico equitativo de la región y a la protección del ambiente local, regional y global a través de la incorporación de criterios de ecoeficiencia, habitabilidad, resil[i]encia, integralidad y viabilidad en la planeación, el diseño, la operación, la rehabilitación y la deconstrucción del ambiente construido... [12, p. 49].

La aplicación del concepto de construcción sostenible en viviendas necesariamente promueve el uso de materiales que minimicen los impactos ambientales, ecológicos, sociales, económicos y tecnológicos negativos causados por los procesos de fabricación, uso y disposición final, a la vez de cumplir también las funciones propias de los materiales de construcción convencionales [13]. Tales materiales son conocidos con el nombre de eco-materiales y se caracterizan, entre otros, por: presentar un bajo consumo energético en las diferentes etapas de fabricación y uso, facilitar un desempeño energético adecuado, emplear recursos y materias primas locales y/o recicladas, ser producidos a partir de tecnologías más amigables con el ambiente y cumplir con los mismos estándares de calidad exigidos a los materiales de construcción convencionales [13].

\section{EL ANÁLISIS DEL CICLO DE VIDA COMO HERRAMIENTA DE EVALUACIÓN DEL DESEMPEÑO AMBIENTAL Y ENERGÉTICO DE LA VIP}

El análisis del ciclo de vida (ACV) es una técnica para evaluar los aspectos e impactos ambientales potenciales de un producto, proceso o sistema a lo largo de toda su cadena de transformación, la cual se basa en la recopilación y análisis de las entradas y salidas del sistema para obtener unos resultados que muestren sus impactos ambientales potenciales, con el objetivo de desarrollar estrategias para su reducción [14]. 
Dentro del ACV los impactos ambientales se agrupan en diferentes categorías, dependiendo del objetivo de estudio y de la exactitud de los resultados requerida. Algunos de las principales categorías de impactos ambientales son: calentamiento global, consumo de recursos energéticos y consumo de materias primas [15]. El sector de la construcción genera impactos significativos en términos de consumo de energía y de recursos, y generación de emisiones. La fabricación de los materiales de construcción necesarios para construir un metro cuadrado de una edificación estándar puede suponer la inversión de una cantidad de energía equivalente a aquella producida por la combustión de más de 150 litros de gasolina. Cada metro cuadrado construido conllevaría entonces una emisión media de 0,5 toneladas de dióxido de carbono y un consumo energético de $1.600 \mathrm{kWh}$ [16].

\section{MEJORA DEL DESEMPEÑO ENERGÉTICO DE LA VIP MEDIANTE EL USO DE LADRILLOS CERÁMICOS MODIFICADOS}

El ladrillo constituye uno de los materiales de construcción más empleados a lo largo de la historia, su uso facilita el cerramiento, la protección y el aislamiento térmico y acústico de las construcciones [17]. Como material de construcción, el ladrillo se puede describir como una pieza paralelepipédica - generalmente en forma de ortoedro- que requiere de procesos de moldeo, secado y cocción de arcillas o tierras arcillosas que se caracterizan por presentar un alto consumo de recursos y energía, y por ende, que causan impactos significativos en el ambiente [18].

Aunque arcilla, agua y calor continúan siendo las principales materias primas en la fabricación del ladrillo, este se empeña en demostrar que la incorporación de materiales tales como los combustibles sólidos recuperados (CSR), mejoran la prestación de propiedades energéticas y su desempeño ambiental, lo cual da origen a los ladrillos modificados. La noción del ladrillo modificado tiene una relación directa con el mejoramiento del desempeño ambiental y energético de las viviendas y edificaciones, así como constituye una oportunidad para reincorporar recursos alternativos provenientes de corrientes residuales y para disminuir los consumos de recursos naturales y energía fósil asociados a los procesos de fabricación de los ladrillos y el uso de la vivienda a lo largo de su ciclo de vida [19].

\section{METODOLOGÍA}

La metodología plantea comparar el desempeño ambiental y energético de ladrillos cerámicos que usan CSR y cenizas volantes de centrales térmicas ,como materiales alternativos que se incorporan a la pasta cerámica en las siguientes proporciones: 
a. Ladrillo cerámico tradicional cocido con carbón (ladrillo 1)

b. Ladrillo cerámico tradicional cocido con CSR (ladrillo 2)

c. Ladrillo cerámico con $7 \%$ de CSR en la pasta cerámica y cocido con este mismo material (ladrillo 3)

d. Ladrillo cerámico con $7 \%$ de CSR y $20 \%$ de cenizas volantes en la pasta cerámica, cocido con combustible sólido recuperado (ladrillo 4)

Para determinar las condiciones de sostenibilidad ambiental se emplearon como metodologías el cálculo del balance energético, el análisis de ciclo de vida y el cálculo de las externalidades ambientales para cada uno de los tipos de ladrillo propuestos, los cuales presentan la composición descrita en la tabla 1 .

Tabla 1. Composiciones de la pasta cerámica para producir una tonelada de ladrillo cerámico cocido

\begin{tabular}{lccccc}
\hline Composición $(\mathrm{kg})^{*}$ & Arcilla & $\begin{array}{c}\text { Cascote de } \\
\text { ladrillo cocido }\end{array}$ & CSR & $\begin{array}{c}\text { Cenizas } \\
\text { volantes }\end{array}$ & Agua \\
\hline Tipo de ladrillo & 1.161 & 132 & - & - & 77 \\
\hline Ladrillo 1 & 1.161 & 132 & - & - & 77 \\
\hline Ladrillo 2 & 1.080 & 132 & 81 & - & 77 \\
\hline Ladrillo 3 & 848 & 132 & 81 & 232 & 77 \\
\hline Ladrillo 4 & & 132 &
\end{tabular}

- En este càlculo se estimó el agua en $\mathrm{kg}$ dado que al considerar su densidad (1,) no afecta el càlculo y favorece el balance de masa e inventario en el ACV.

Fuente: elaboración propia

\subsection{Desempeño energético}

En el proceso de producción de ladrillos cerámicos intervienen dos tipos de energía: térmica y eléctrica. Este ejercicio de investigación solo considera el balance de energía térmica y parte del presupuesto de que el proceso cerámico no presentará cambios de masa representativos puesto que la arcilla -principal materia prima en la producción de ladrillos cerámicos- contiene bajos porcentajes de materia orgánica y el calor de descomposición, asociado al agua de constitución y a las sales susceptibles de descomposición, hacen parte del calor de reacción. Además del calor de reacción, en el balance energético (energía consumida) se tuvo en cuenta el calor de calentamiento, las pérdidas de calor a través de paredes y el calor sensible de los humos, como se muestra en la ecuación 1 .

Ecuación 1. Balance asociado a la energía térmica en la producción de ladrillos cerámicos

Energía consumida $=$ calor calentamiento + calor reacción + calor sensible + pérdidas 
El calor de calentamiento hace referencia a la acumulación de calor de la pasta cerámica a cocer, para lo cual se emplea la ecuación 2. Los resultados se presentan en la sección 5.1, en la tabla 3.

Ecuación 2. Cálculo del calor de calentamiento

$$
\text { Calor de calentamiento }=\mathrm{Kg}_{\text {arcilla }} * \text { Calor específico } \frac{\mathrm{Kcal}}{\mathrm{Kg}_{\text {arcilla }}{ }^{\circ} \mathrm{C}} * \text { Temperatura de quema }\left({ }^{\circ} \mathrm{C}\right)
$$

Para el cálculo del calor de reacción se considera la energía térmica como la base de las reacciones físico-químicas que constituyen la esencia de la cocción cerámica. Tales reacciones se dan a altas temperaturas $\left(55-800^{\circ} \mathrm{C}\right)$; las reacciones de pérdida de agua de cristalización de los minerales de arcilla son las que absorben mayor calor. Se emplea la ecuación 3, que supone un $8 \%$ de agua de constitución contenida en los minerales de arcilla y un requerimiento energético de $600 \mathrm{Kcal} / \mathrm{Kg} \mathrm{H}_{2} \mathrm{O}$. Los resultados se presentan en la sección 5.1, tabla 3.

Ecuación 3. Cálculo del calor de reacción

$$
\text { Calor de reacción }=\mathrm{Kg}_{\text {arcilla }} * \frac{\mathrm{Kg} \mathrm{H}_{2} \mathrm{O}}{\mathrm{Kg}_{\text {arcilla }}} * \frac{\mathrm{Kcal}}{\mathrm{Kg} \mathrm{H}_{2} \mathrm{O}}
$$

El calor sensible de los humos es una función del caudal de gases, del calor específico de los gases y del gradiente de temperatura entre la salida y el ambiente externo. Se emplea un calor sensible de $120 \mathrm{Kcal} / \mathrm{kg}$ de cerámica cocida. Finalmente, la pérdida de calor a través de paredes es una variable que depende tanto de condiciones y características de los hornos, como del tipo de horno y el aislamiento térmico con el que cuenta, de las condiciones de combustión, así como de la circulación y velocidad de los gases. Se emplearon pérdidas de calor a través de paredes de 70 y $66,5 \mathrm{Kcal} / \mathrm{kg}$ de cerámica cocida, ver tabla 3 .

\subsection{Desempeño ambiental}

El desempeño ambiental de los ladridos se mide con la herramienta de análisis del ciclo de vida (ACV) propuesta -en la cumbre mundial para el Desarrollo Sustentable de Johannesburgo en el 2002- como un modelo para "mejorar los productos y servicios a la vez que se reducen los impactos en salud y medio ambiente" [20]. El ACV es una metodología que, además de permitir la integración de los impactos ambientales ocurridos a lo largo del ciclo de vida, los relaciona con problemas ambientales específicos; permite establecer una unidad de medida ambiental basado en los distintos problemas ambientales y establecer prioridades ambientales que permiten planificar el mejoramiento del desempeño ambiental de un producto o servicio [20]. 
El ACV propuesto en esta investigación se planteó con base en los lineamientos de la norma ISO 14040 [21], la cual se enmarca en el proceso productivo de ladrillos cerámicos para su uso en el proyecto de VIP Tirol III, Megaproyecto Pajarito, desarrollado por Isvimed en la ciudad de Medellín; se consideraron las siguientes etapas: extracción y preparación de la arcilla, conformación, corte, secado, cocción y distribución del ladrillo a la obra. De igual manera, se estableció como unidad funcional 1 y $0,92 \mathrm{Kg}$ de arcilla cocida, según el tipo de ladrillo cerámico (ver tabla 2), estos los produce una ladrillera con horno tipo Hoffman.

Tabla 2. Unidad funcional para el ACV, según tipo de ladrillo cerámico

\begin{tabular}{cc}
\hline Tipo de ladrillo cerámico & Unidad funcional $(\mathrm{Kg})$ \\
\hline Ladrillo 1 & 1 \\
\hline Ladrillo 2 & 1 \\
\hline Ladrillo 3 & 0,92 \\
\hline Ladrillo 4 & 0,92 \\
\hline
\end{tabular}

Fuente: elaboración propia

El inventario del sistema tuvo en cuenta las entradas en términos de consumos de materiales y energía/combustibles, así como las salidas en términos de emisiones de dióxido de carbono, material particulado, vapor de agua, dióxido de azufre, nitrógeno y oxigeno; y otros impactos asociados a la minería de arcillas. Tales datos se tradujeron en puntos de impacto ambiental mediante el empleo de 11 categorías establecidas en la metodología Eco Indicador 99, con la base de datos Ecoinvent V2.2 y el software Simapro V7.2.

Dentro del inventario global del proceso de fabricación de ladrillos cerámicos modificados a partir del uso de CSR como elemento constituyente de la pasta cerámica y/o como combustible alternativo empleado en los procesos de secado y cocción de este, se ha de destacar un importante impacto positivo: el metano que se evita por la disposición del CSR en rellenos sanitarios. Para estimar este impacto se empleó el modelo de decaimiento exponencial de primer orden requerido por la herramienta Tool to determine methane emissions avoided from dumping waste at a solid waste disposal site, versión 2, para proyectos de Mecanismos de Desarrollo Limpio. En esta ecuación se consideró una tasa de decaimiento del CSR de 0,03 y un CSR con 33,80\% de carbono orgánico.

\subsection{Externalidades}

La teoría de las externalidades hace referencia a todos los costos y beneficios que recaen sobre la sociedad y el medio ambiente como consecuencia de una actividad 
económica, los cuales se caracterizan por no estar incluidos en el precio del bien o servicio que los genera; así, estos no interfieren en los costos y beneficios de quien los produce, pero sí suponen un coste social, materializado generalmente en impactos ambientales y/o socioeconómicos [22].

En esta investigación se consideraron los siguientes beneficios sociales y ambientales derivados de la fabricación y el uso de ladrillos modificados:

- Disminución de efectos en la población asociados a la reducción de emisiones de dióxido de carbono.

- Disminución del agotamiento de recursos naturales no renovables y mayor disponibilidad para la población por la reducción del consumo de arcilla.

- Disminución del agotamiento de recursos naturales no renovables y mayor disponibilidad para la población por la reducción del consumo de combustibles fósiles.

Se asume un proceso productivo para una producción mensual de 50.000 ladrillos cerámicos, en el que se explota arcilla hasta 30 metros de profundidad, con un costo de extracción de $\$ 4.500 /$ tonelada y un costo de transporte de $\$ 2.500 /$ tonelada; proceso en el que además se destinan $\$ 250$ por tonelada extraída para la restauración de las áreas de explotación.

\section{$5 \quad$ RESULTADOS}

\subsection{Desempeño energético}

Usando la ecuación 1 y los datos presentados en la tabla 3, el balance energético para los diferentes tipos de pasta cerámica se presenta en la tabla 4 .

Tabla 3. Requerimientos de energía térmica en la producción de ladrillos cerámicos

\begin{tabular}{|l|c|c|c|c|}
\hline $\begin{array}{c}\text { Tipo de ladrillo } \\
\text { cerámico }\end{array}$ & $\begin{array}{c}\text { Calor de } \\
\text { calentamiento } \\
\text { (Kcal) }\end{array}$ & $\begin{array}{c}\text { Calor de reacción } \\
\text { (Kcal) }\end{array}$ & $\begin{array}{c}\text { Calor sensible de } \\
\text { los humos } \\
\text { (Kcal) }\end{array}$ & $\begin{array}{c}\text { Pérdidas de } \\
\text { calor a través de } \\
\text { paredes (Kcal) }\end{array}$ \\
\hline Ladrillo 1 & 278,64 & 55,73 & 120 & 70 \\
\hline Ladrillo 2 & 278,64 & 55,73 & 120 & 70 \\
\hline Ladrillo 3 & 259,2 & 51,84 & 120 & 70 \\
\hline Ladrillo 4 & 203,52 & 40,70 & 120 & 66,5 \\
\hline
\end{tabular}


Tabla 4. Balance energético y consumo de combustible (CSR) para cada tipo de ladrillo cerámico

\begin{tabular}{lcc}
\hline Tipo de ladrillo & $\begin{array}{c}\text { Balance energético } \\
(\text { Kcal })\end{array}$ & $\begin{array}{c}\text { Consumo de CSR (Kg } \\
\text { CSR/ Kg arcilla) }\end{array}$ \\
\hline Ladrillo 1 & 524,37 & 0,150 \\
\hline Ladrillo 2 & 524,37 & 0,150 \\
\hline Ladrillo 3 & 501,04 & 0,143 \\
\hline Ladrillo 4 & 420,55 & 0,123 \\
\hline \multicolumn{3}{c}{ Fuente: elaboración propia }
\end{tabular}

\subsection{Desempeño ambiental}

Al aplicar el modelo de decaimiento exponencial de primer orden con la herramienta Tool to determine methane emissions avoided from dumping waste at a solid waste disposal site, versión 2 para proyectos de Mecanismos de Desarrollo Limpio, se obtiene el volumen de metano evitado de acuerdo con el tipo de ladrillo cerámico modificado propuesto que aparece en la tabla 5.

Tabla 5. Metano evitado por el uso de CSR en el proceso de fabricación de ladrillo cerámico como materia prima de la pasta cerámica y/o como combustible

\begin{tabular}{lcc}
\hline Tipo de ladrillo cerámico & CSR empleado $(\mathrm{Kg})$ & $\begin{array}{c}\text { Metano evitado } \\
\text { (Kg CH4/Kg CSR })\end{array}$ \\
\hline Ladrillo 1 & - & - \\
\hline Ladrillo 2 & 0,13657 & $4,201 \mathrm{E}-04$ \\
\hline Ladrillo 3 & 0,21263 & $6,541 \mathrm{E}-04$ \\
\hline Ladrillo 4 & 0,20515 & $6,320 \mathrm{E}-04$ \\
\hline
\end{tabular}

Fuente: elaboración propia

La tabla 6 y la figura 1 muestran el desempeño ambiental de cada uno de los ladrillos cerámicos propuestos para esta investigación; este se mide en términos de puntos de impacto ambiental para cada una de las 11 categorías establecidas en la metodología Eco Indicador 99: 
Tabla 6. Datos del desempeño ambiental de un ladrillo cerámico tradicional con ladrillos cerámicos modificados a partir del uso de CSR en la pasta cerámica y/o como combustibles

\begin{tabular}{|c|c|c|c|c|c|}
\hline Categoría de impacto & Unidad & $\begin{array}{c}\text { Ladrillo } \\
\text { cerámico } \\
\text { tradicional } \\
\text { cocido con } \\
\text { carbón }\end{array}$ & $\begin{array}{l}\text { Ladrillo cerámico } \\
\text { tradicional } \\
\text { cocido con CSR }\end{array}$ & $\begin{array}{c}\text { Ladrillo } \\
\text { cerámico con } \\
7 \% \text { de CSR } \\
\text { en la pasta } \\
\text { cerámica y } \\
\text { cocido con este } \\
\text { mismo material }\end{array}$ & $\begin{array}{c}\text { Ladrillo cerámico } \\
\text { con } 7 \% \text { de CSR, } \\
20 \% \text { de cenizas } \\
\text { volantes en la pasta } \\
\text { cerámica, cocido con } \\
\text { combustible sólido } \\
\text { recuperado }\end{array}$ \\
\hline Total & $\mathrm{Pt}$ & 0,020724 & 0,01975 & 0,01529 & 0,01488 \\
\hline Cancerígenos & $\mathrm{Pt}$ & 0,000976 & 0,00025 & 0,00022 & 0,00020 \\
\hline Respirables orgánicos & $\mathrm{Pt}$ & 0,000005 & 0,00000 & 0,00000 & 0,00000 \\
\hline Respirables inorgánicos & $\mathrm{Pt}$ & 0,002764 & 0,00262 & 0,00192 & 0,00185 \\
\hline Cambio climático & $\mathrm{Pt}$ & 0,005588 & 0,00478 & 0,00345 & 0,00359 \\
\hline Radiación & $\mathrm{Pt}$ & 0,000003 & 0,00000 & 0,00000 & 0,00000 \\
\hline Capa de ozono & $\mathrm{Pt}$ & 0,000000 & 0,00000 & 0,00000 & 0,00000 \\
\hline Eco toxicidad & $\mathrm{Pt}$ & 0,000366 & 0,00019 & 0,00018 & 0,00015 \\
\hline Acidificación/Eutrofización & $\mathrm{Pt}$ & 0,000379 & 0,00039 & 0,00029 & 0,00028 \\
\hline Usos del suelo & $\mathrm{Pt}$ & 0,000621 & 0,00063 & 0,00035 & 0,00038 \\
\hline Minerales & $\mathrm{Pt}$ & 0,000068 & 0,00007 & 0,00006 & 0,00006 \\
\hline Combustibles fósiles & $\mathrm{Pt}$ & 0,009955 & 0,01081 & 0,00882 & 0,00835 \\
\hline
\end{tabular}

Fuente: elaboración propia

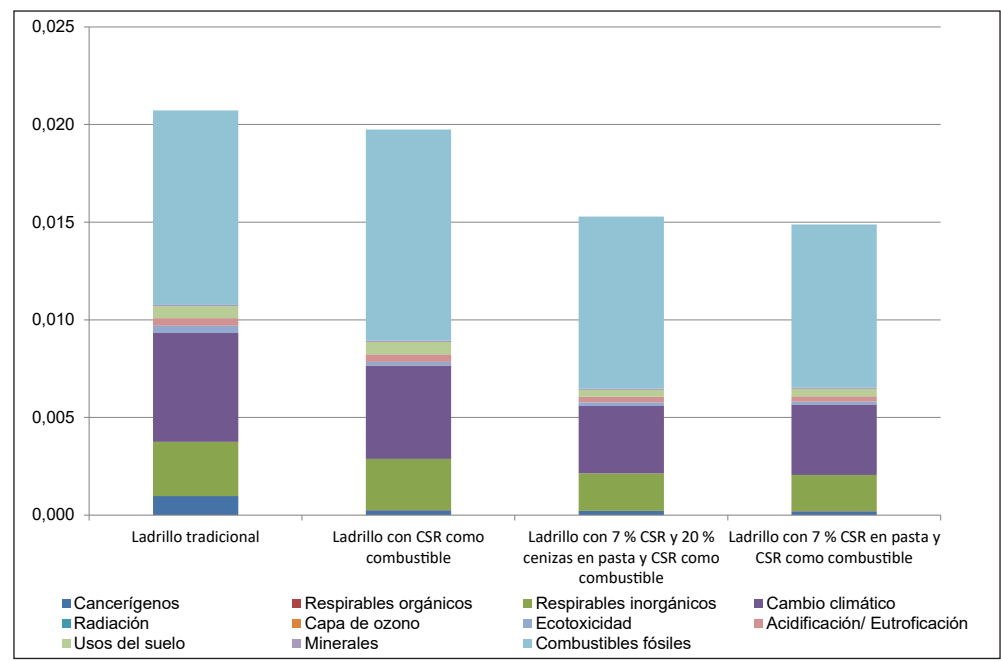

Figura 1. Comparativo del desempeño ambiental de un ladrillo cerámico tradicional con ladrillos cerámicos modificados con el uso de CSR en la pasta cerámica y/o como combustibles

Fuente: elaboración propia con base en datos de la Tabla 6 


\subsection{Externalidades}

\subsubsection{Disminución de emisiones de dióxido de carbono}

El uso de CSR como combustible y como parte de la pasta cerámica contribuye a la reducción de las emisiones de dióxido de carbono que ocurren en los procesos tradicionales de secado y cocción del ladrillo. La tabla 7 muestra las emisiones de dióxido de carbono reducidas (ton $\mathrm{CO} 2$ eq) y los costos evitados por cambio climático, el considerar que el Panel Intergubernamental de Expertos en Cambio Climático del 2006 estableció que los gobiernos deberían invertir \$ 50 USD por tonelada de dióxido de carbono emitida.

Tabla 7. Emisiones de dióxido de carbono equivalente y costos evitados por cambio climático

\begin{tabular}{lcc}
\hline Descripción & $\begin{array}{c}\text { Emisiones reducidas } \\
\text { (Ton } \mathrm{CO}_{2} \text { eq/año) }\end{array}$ & $\begin{array}{c}\text { Costos evitados por cambio climático } \\
\text { (\$ USD/año) }\end{array}$ \\
\hline Ladrillo 1 & 0,00 & 0 \\
\hline Ladrillo 2 & 859 & 42,974 \\
\hline Ladrillo 3 & 695 & 34,757 \\
\hline Ladrillo 4 & 552 & 27,604 \\
\hline
\end{tabular}

Fuente: elaboración propia

\subsubsection{Disminución del consumo de arcilla}

La extracción y transporte de arcilla, así como los procesos de restauración asociados a la recuperación de las áreas degradadas y/o intervenidas por la su extracción conllevan la generación de costos que se podrían reducir a través de la sustitución de la arcilla por materiales alternativos en la pasta cerámica, como es el caso del CSR propuesto en esta investigación. La tabla 8 presenta los ahorros asociados a los volúmenes de arcilla reducidos en términos de explotación y transporte, y a la restauración de las áreas degradadas por el proceso de minería de esta.

Tabla 8. Ahorros por explotación y transporte de la arcilla extraída y por restauración de áreas de extracción de arcilla

\begin{tabular}{|l|c|c|c|}
\hline Descripción & $\begin{array}{c}\text { Volumen de arcilla } \\
\text { reducida (ton/año) }\end{array}$ & $\begin{array}{c}\text { Ahorro en costos explotación } \\
\text { y transporte (\$/año) }\end{array}$ & $\begin{array}{c}\text { Ahorro en costos de } \\
\text { restauración (\$/año) }\end{array}$ \\
\hline Ladrillo 1 & 0 & 0 & 0 \\
\hline Ladrillo 2 & 0 & 0 & 0 \\
\hline Ladrillo 3 & 783 & 5.484 .254 & 195.866 \\
\hline Ladrillo 4 & 1.465 & 10.258 .483 & 366.374 \\
\hline
\end{tabular}




\subsubsection{Disminución del consumo de combustibles fósiles}

El uso de combustibles fósiles, como el carbón, en los procesos de producción de ladrillos cerámicos, conlleva a la generación de costos que son contabilizados directamente en su proceso de producción. De igual manera, existen algunos costos que no afectan de forma directa la producción, como son los costos de la explotación del carbón y los costos asociados a los procesos de restauración de áreas de extracción. La tabla 9 describe los ahorros asociados a la sustitución de este por combustibles alternativos, como el CSR.

Tabla 9. Ahorros asociados al consumo de carbón

\begin{tabular}{lcc}
\hline Descripción & $\begin{array}{c}\text { Reducción consumo de } \\
\text { carbón Ton/año }\end{array}$ & $\begin{array}{c}\text { Ahorro costos consumo de } \\
\text { carbón \$ COP/año }\end{array}$ \\
\hline Ladrillo 1 & 0 & 0 \\
\hline Ladrillo 2 & 391 & 58.600 .451 \\
\hline Ladrillo 3 & 316 & 47.396 .119 \\
\hline Ladrillo 4 & 251 & 37.642 .368 \\
\hline \multicolumn{3}{c}{} \\
\hline
\end{tabular}

\section{CONCLUSIONES}

- Si bien el sector de las edificaciones es uno de los que más contribuye al cambio climático mediante la generación de un porcentaje considerable de las emisiones globales de gases de efecto invernadero y altos consumos de recursos y energía a lo largo de su ciclo de vida, también es uno de los sectores con mayor potencial para reducir estas emisiones y el consumo de recursos y energía. De ahí que la adopción de principios y conceptos de edificaciones sostenibles -como el uso de ladrillos modificados- que estimulen el mejoramiento del desempeño ambiental y energético a lo largo del ciclo de vida de las edificaciones, se presenta como una alternativa para promover y aprovechar dicho potencial.

- Al evaluar el desempeño energético de VIP que emplean ladrillos tradicionales y ladrillos modificados en su proceso constructivo se encontró que las VIP que emplean ladrillos modificados presentan requerimientos energéticos y por tanto generan una huella de carbono considerablemente menor, entre un 4 y un $9 \%$ menos en comparación con un VIP que emplea ladrillos tradicionales.

- Al emplear ACV como herramienta para medir el desempeño ambiental de ladrillos tradicionales y ladrillos modificados se encuentra que la reducción de impactos ambientales se aproxima al $25 \%$ con relación al ladrillo tradicional, cuando se 
emplean ladrillos modificados y procesados con combustibles renovables. Destaca la reducción de impactos asociados al consumo de recursos (combustibles fósiles) y efectos asociados con el cambio climático.

- Los ladrillos modificados propuestos en este trabajo corresponden a tres tipologías: la primera, un ladrillo tradicional cocido con CSR; el segundo, un ladrillo con sustitución del $7 \%$ de arcilla por CSR y cocido con CSR; y por último, un ladrillo con sustitución del 7 y $20 \%$ de arcilla con CSR y ceniza volante respectivamente, cocido con CSR. Estas modificaciones proponen un ahorro en el consumo de recursos naturales no renovables como la arcilla y el carbón que tradicionalmente se emplean en esta industria. Asimismo, se promueve la recuperación, reciclaje y reintegración de recursos alternativos provenientes de fuentes secundarias, como en el caso de los residuos sólidos urbanos considerados en el presente trabajo.

- Es posible explorar otros recursos alternativos con presentaciones semejantes en otros trabajos, de esta manera, la estrategia de empleo de ladrillos modificados en las VIP contribuiría igualmente con la reducción de problemas de carácter ambiental asociados a la gestión, el tratamiento y la eliminación de residuos urbanos e industriales, mientras se optimiza el aprovechamiento y uso de recursos no renovables.

- La propuesta de empleo de ladrillos modificados genera un conjunto de externalidades entre las que destacan innovación y desarrollo tecnológico, nuevos procesos industriales y modelos de negocio, mayor rentabilidad y competitividad empresarial, responsabilidad social, disminución en la generación de impactos ambientales como contaminación del aire efectos en la salud pública asociados.

- Aunque en el contexto internacional el uso de ladrillos modificados ya se encuentra inserto en el mercado de la construcción, el desarrollo de estos en Medellín y en Colombia es, por el momento, una propuesta investigativa. Aún cuando ha sido conocida y probada de manera suficiente en el sector ladrillero y de la industria de la construcción, la actual normatividad técnica colombiana para este tipo de productos restringe la inserción de materiales sustitutos en la producción de los ladrillos, a pesar de ser un hecho técnico y económicamente viable.

- Las VIP de la ciudad son promovidas principalmente por las agencias del gobierno local, en coordinación con las directrices nacionales. La concepción de proyectos de VIP sigue criterios y parámetros muy convencionales que no dan pie a, ni promueven la adopción de este tipo de innovaciones. Se evidencia una falta de conocimiento en los equipos de diseño y gestión de proyectos de estas 
entidades, limitando la inserción de soluciones sostenibles e innovadoras probadas $\mathrm{y}$ adoptadas suficientemente en el contexto internacional.

- Es imperativo reestructurar la normatividad técnica del ladrillo con el fin de posibilitar el uso de ladrillos modificados en la construcción, no solo de VIP sino en edificaciones en general. Esto se puede hacer inicialmente para ladrillos no estructurales o portantes. Igualmente es necesario elevar el nivel de conocimiento de diseñadores, arquitectos y funcionarios responsables de este tipo de obras.

\section{REFERENCIAS}

[1] S. Solís San Vicente, "Las necesidades sociales: el acercamiento a su construcción," en $\mathrm{Ne}$ cesidades sociales y desarrollo humano: un acercamiento metodológico, C. Arteaga-Basurto y S. Solís-San Vicente, eds., Barcelona: Plaza y Valdés, pp. 27-70, 2005.

[2] Alcaldía Distrital de Bogotá, Comité de Hábitat, "Política de hábitat y seguridad humana. Documento interno de discusión”, inédito, 2003.

[3] "Parlamento Andino", III Cumbre Social Andina - Vivienda Social, [En línea], acceso 03 de octubre. Disponible: https://biblioteca-parlamentoandino.janium.net/janium-bin/pdfview.pl $? \mathrm{Id}=20190910160948 \& \mathrm{r}=5485 \& \mathrm{t}=\mathrm{p}, 2013$.

[4] Ministerio de Vivienda, Ciudad y Territorio, Decreto 0075. Por el cual se reglamentan el cumplimiento de los porcentajes de suelos destinados a programas de Vivienda de Interés Social para precios sujetos a los tratamiento urbanísticos de desarrollo y renovación y se dictan otras disposiciones. [En línea], Disponible: http://www.minvivienda.gov.co/Decretos\%20Vivienda/0075\%20-\%20.pdf, 2013.

[5] Ministerio de Ambiente, Vivienda y Desarrollo Territorial (MAVDT), Serie Guías de Asistencia Técnica para Vivienda de Interés Social - 1. Calidad en Vivienda de Interés Social. Bogotá: Nuevas Ediciones SAS, 2011.

[6] Área Metropolitana del Valle de Aburrá (AMVA) y Universidad Pontificia Bolivariana (UPB), Política Pública de Construcción Sostenible: 1. Línea base, Medellín: AMVA y UPB, 2015.

[7] Alcaldía de Medellín, Plan Estratégico Habitacional de Medellín, Pehmed 2020: Medellín mi casa 2020: Una cuidad pensada y construida por todos [Síntesis de Pehmed 2020], Medellín: Alcaldía de Medellín, Instituto Social de Vivienda y Hábitat de Medellín (Isvimed), Universidad Nacional, Escuela del Hábitat (Cehap), Corporación Viva la Ciudadanía, 2011.

[8] Instituto Social de Vivienda y Hábitat de Medellín (Isvimed), “Tus sueños ya tienen donde vivir,” Medellín: Isvimed. [En línea], Disponible: https://www.youtube.com/ watch?v=hGOMWSV9oLQ, 2014.

[9] "The United Nations Environment Programme Sustainable Buildings \& Climate ChangeUNEP,” Building and Climate Change Summary for Decision-Makers, Francia: UNEP DTIE. 
[En línea], Disponible: https://europa.eu/capacity4dev/unep/document/buildings-and-climate-change-summary-decision-makers, 2009.

[10] A. Hoballah, "Building Design and Construction: Forcing Resource Efficiency and Sustainable Development", The United Nations Environment Programme Sustainable Buildings \& Climate Change - UNEP,” Francia: UNEP DTIE, Reporte técnico, [En línea], Disponible: https://www.usgbc.org/ShowFile.aspx?DocumentID=19073, 2012.

[11] P. Alavedra, J. Domínguez, E. Gonzalo, y J. Serra, "La construcción sostenible: el estado de la cuestión. Informes de la Construcción," Informes de la Construcción, vol. 49, $\mathrm{n}^{\circ}$ 451[En línea], Disponible: http://informesdelaconstruccion.revistas.csic.es/index.php/ informesdelaconstruccion/article/view/936/1018

[12] Área Metropolitana del Valle de Aburrá (AMVA) y Universidad Pontificia Bolivariana (UPB), Política Pública de Construcción Sostenible: 3. Lineamientos, Medellín: AMVA y UPB, 2015, 49 p.

[13] R. Bancrofft, Ecomateriales de construcción: una contribución a la salud y a la vivienda, Ciudad de la Habana, Cuba: Instituto Superior Politécnico José A. Echeverría (ISPJAE), [En línea], Disponible: http://www.bvsde.paho.org/vivi/cd/inhem2/reunion/tccomat.htm, 1997.

[14] Instituto Colombiano de Normas Técnicas y Certificación, Gestión Ambiental. Análisis de ciclo de vida. Principios y narco de referencia, Reporte NTC-ISO 14040, Colombia, 2006

[15] Ihobe, Sociedad Pública de Gestión Ambiental, Análisis de ciclo de vida y huella de carbono: dos maneras de medir el impacto ambiental de un producto, Bilbao: Ihobe, Sociedad Pública de Gestión Ambiental, 2009, pp. 3-25.

[16] A. Aranda-Usón, I. Zabalza-Bribián, S. Díaz de Garayo, y S. Scarpellini, “Impactos de los materiales de construcción, análisis de ciclo de vida," Revista EcoHabitar, [En línea], Disponible: http://www.ecohabitar.org/impacto-de-los-materiales-de-construccion-analisis-deciclo-de-vida/, 2014.

[17] F. Vela-Cossio, J. De Hoz-Onrubia, y L. Maldonado-Ramos, Diccionario de construcción tradicional tierra, San Sebastián: Editorial Nerea SA, 2003.

[18] F. Mingarro-Martín, Curso de verano en el Escorial: degradación y conservación del patrimonio arquitectónico, Madrid: Editorial Complutense, 1996.

[19] X, Elías, Reciclaje de residuos industriales, residuos sólidos urbanos y fangos de depuradora, 2a ed., Madrid: Ediciones Díaz de Santos SA, 2009.

[20] A. Hoof, y N. Suppen, "Conceptos básicos de análisis de ciclo de vida y su aplicación en el ecodiseño", Centro de Análisis de Ciclo de Vida y Diseño Sustentable, [En línea], Disponible: https://nanopdf.com/queue/manual-centro-regional-de-produccion-mas-limpia_pdf?queue_ $\mathrm{id}=-1 \& \mathrm{x}=1568150865 \& \mathrm{z}=\mathrm{MTkwLjAuMi} 4 \mathrm{xMjY}=, 2005$. 
[21] ISO, “Gestión Ambiental. Análisis de Ciclo de Vida. Requisitos y Directrices. Requisitos del Ciclo de Vida," Colombia, Reporte NTC ISO 14044, 2007.

[22] P. Martinez de Anguita, “Economía ambiental y ordenación del territorio," Revista ecosistemas, vol. 13, n. ${ }^{\circ} 1,2004$, pp. 87-93. 